shows that a paradox of negative energy levels disappears $^{1}$. It seems in any case significant that the order of these uncertainties is that at which it is known that wave mechanies begins to break down.

A further conceivable development of the argument is its connexion with changes associated with a direction of time. If recognizable changes of con. figuration could be imagined referred to an ideal time defined in terms of the number of interactions that take place, then as entropy increases, the approach of equilibrium will imply that more interactions will take place before a recognizable change occurs. If our observable time is regarded as dependent on recognizable change, then, being a statistical average, it will as a macroscopic phenomenon eventually disappear. Before this stage is reached, however, it will in terms of our ideal time go more and more slowly; and it could appear to follow, if we adopt conventionally a fixed relation between measurements in space and time (denoted by the constant $c$ ), that space will correspondingly expand. Equivalently, the velocity of light could be said to decrease, though it should be noted, in view of past suggestions (on rather insufficient evidence), that this velocity is apparently decreasing, that even the present observed expansion of space, whatever its cause, would, on the assumption of an absolute constant for the velocity, only give rise to an apparent decrease of about $16 \mathrm{~cm}$./sec./year, which would be undetectable ${ }^{2}$.

In spite of possible fallacies in the above arguments, they may serve to stress properties of an observable time that are now perhaps becoming appreciated. Thus it should be remembered that a clock is not merely an oscillating system that is observed at a particular instant-this would define in terms of space a periodic sort of time; if it has also to record its number of oscillations without disturbance, it begins to be a more complicated mechanism.

Jealott's Hill Research Station,

M. S. Bartlett.

Bracknell, Berks.

August 6.

1 Nature, 137, 313 (1936).

2 ef. Edmondson, NATURE, 133, 759 (1934).

\section{Genotypic Control of Chromosome Size}

THE differences in size between the chromosomes of related species are usually differences that can be attributed to structural changes such as fusion, fragmentution, etc. In many groups, however, enormous differences of size are found, which may be of the order of $1: 100$ or even $1: 1,000$. Such differences, uniformly affecting as they often do the whole complement, must be due to a unitary genotypic control of chromosome size. In other words, the size of chromosomes, like other properties of the organism, must be subordinate to the action of the genes they carry ${ }^{1}$.

Experimental examples of this principle are very rare, the only clear case being that of a bud sport in a triploid plant presumed to be a hybrid between diploid and tetraploid Tradescantia species with different chromosome sizes. The mutant had chromosomes $1 / 5$ the size of the stock $^{2}$.

In experimental material of grasses $^{3}$, kindly supplied by Dr. T. J. Jenkin, of the Welsh Plant Breeding Station, Aberystwyth, I have been able to find evidence of the inheritance of this genotypic property governing chromosome size. A male sterile plant of Lolium perenne (Fig. 1,A), with chromosomes of about a quarter the size of the normal, was crossed with a normal plant and gave 8 male sterile seedlings, two of which were examined. One of them (Fig. 1, B) proved to have chromosomes of normal size for the species. The other (Fig. 1, $C$ ) has chromosomes of more than twice this size.

I have found similar differences in crosses between Festuca arundinacea and $F$. pratensis4.

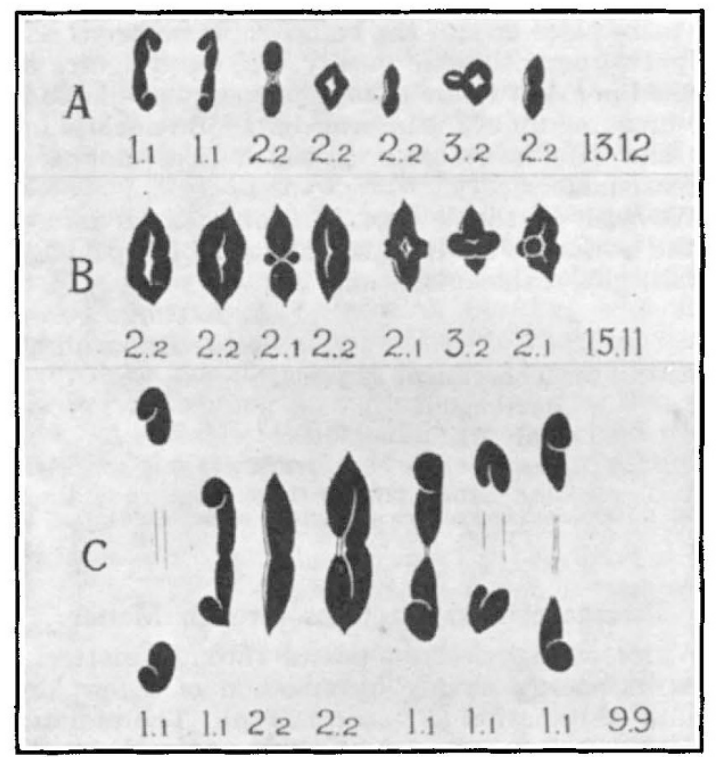

FIG. 1. Metaphase and early anaphase bivalents in three Lolium perenne plants. $A$ is the female parent of both $B$ and $C$. Total and terminal chiasmata are given under each bivalent. Acetocarmine preparations. $\times$ about 1,700 .

Further study will be necessary to determine the mode of inheritance of this character, but the present observations prove that it is subject to Mendelian segregation. They therefore throw doubt on statements that characteristic chromosome size differences between species are maintained in their hybrids. Some of these statements have already been disproved $^{5}$.

John Innes Horticultural Institution,

Percy T. Thomas. London, S.W.19. August 5.

1 Darlington, Amer. Nat., 66, 25 (1932).

8 Darlington, J. Genet., 21, 207 (1929).

${ }^{3}$ Jenkin, Welsh Plant Breeding Station Bull., H.12 (1931).

Jenkin, J, Genet., 28, 205 (1934).

Upcott, $J$. Genet., (in the press).

\section{Behaviour of Local Drosophila melanogaster during Late Larval Stage}

During March 1935 a few local Drosophila flies which emerged from the soil and leaves of a millipede culture of Dr. N. F. Paterson were collected, and a culture was started. The stock proved to be of good viability; the flies were identified as Drosophila melanogaster. After the cultures had been kept for a few generations it was observed that the larvæ of this 'Witwatersrand' stock displayed an interesting difference in behaviour compared with the standard D. melanogaster cultures. During their late larval 